\title{
A New Type of Generalized F-Expansion Method and its Application to Sine-Gordon Equation
}

\author{
Yusuf Pandir \\ Bozok University, Faculty of Arts and Sciences, Department of Mathematics, Yozgat, Turkey, +903542421021, \\ yusuf.pandir@bozok.edu.tr
}

Received: 24 March 2017

Accepted: 17 May 2017

DOI: $10.18446 /$ cbayarfbe.306899

\begin{abstract}
In this article, a new type of generalized F-expansion method which is very distinct implementation is proposed. Combined and multiple Jacobi elliptic functions solutions are presented with this suggested method. Analytical solutions of sine-Gordon equation are found by using the new type of generalized F-expansion method. As a result, many new and more general function solutions are acquired such as single, combined and multiple nondegenerate Jacobi elliptic function solutions.

Keywords - A new type of generalized F-expansion method, Sine-Gordon equation, Combined Jacobi elliptic functions solutions, Multiple Jacobi elliptic functions solutions.
\end{abstract}

\begin{abstract}
1 Introduction
Understanding the laws that exist in nature has caused physical problems to arise. The suggested method for solving these types of problems has gain meaning by using mathematical theories. In general, problems arising in many branches of applied science have been expressed by using mathematical models. A mathematical model is designed to explain a system and provide information about its behavior. Therefore studies on solutions of nonlinear partial differential equations have become more significant. Especially, many scientists who seeking exact solutions for nonlinear partial differential equations have focused on new analytical methods. When we look at recent studies, we see that many researchers offer different methods such as $G^{\prime}$ / $G$-expansion method [1], exp-function method [2], Hirota bilinear method [3], trial equation method [4], Lie symmetry analysis method [5], extended trial equation method [6], Jacobi elliptic function method [7], Weierstrass elliptic function expansion method [8], F-expansion method [912], generalized Kudryashov method [13] and modified Kudryashov method [14] to find exact solutions of the nonlinear differential equations. It is very important to achieve the Jacobi elliptic function solutions of nonlinear partial differential equations. One of the analytical methods that give Jacobi elliptic function solution is the F-expansion method. The F-expansion method has more than one type. Methods that allow us to find Jacobi elliptic function solutions are methods such as the F-expansion method, the improved Fexpansion method [15], extended F-expansion method [16]
\end{abstract}

and the generalized F-expansion method $[17,18]$. In the present paper, a more general case of these methods is introduced as a new method by looking at different types of Fexpansion method from considers a different point of view. This suggested method will give us to find combined and multiple Jacobi elliptic functions together in the solution function. New and different Jacobi elliptic function solutions have been obtained by this new method.

The rest of this paper is regulated as follows: in Section 2, developed new type of generalized F-expansion method for partial differential equations has been introduced. In Section 3, the new exact analytical solutions to sine-Gordon equation have investigated by use of the new type of generalized F-expansion method. Finally, the conclusions have given in Section 4.

\section{New type of generalized F-expansion method}

In this section, a new type of generalized F-expansion method which is not available in the literature is introduced. For the given general nonlinear differential equation of the type.

$$
G\left(u, u_{x}, u_{y}, u_{t}, \ldots, u_{x x}, u_{x y}, u_{x t}, \ldots\right)=0
$$

where $u(x, y, t, \ldots)$ is an unknown function, $x, y, t, \ldots$ independent variables (space coordinates) and $G$ is a polynomial of $u$ and its partial derivatives, in which the highest order derivatives and the nonlinear terms are included. 
When we implemented the following traveling wave transformation to Eq. (1),

$$
u(x, y, t, \ldots)=U(\xi), \quad \xi=a x+b y+c t+\ldots,
$$

which $a, b$ and $c$ are constants to be determined later, we reduced Eq. (1) to nonlinear ordinary differential equation,

$$
H\left(U, U^{\prime}, U^{\prime \prime}, \ldots\right)=0,
$$

where the prime denotes the derivation with respect to $\xi$. Let suppose that a solution in the following form of the Eq. (3),

$$
U(\xi)=a_{0}+\sum_{i=1}^{N} a_{i} F^{i}+\frac{b_{i}}{F^{i}}+c_{i}\left(\frac{F^{\prime}}{F}\right)^{i}+d_{i}\left(\frac{F}{F^{\prime}}\right)^{i}
$$

in which $F=F(\xi)$ and $\quad F^{\prime}=F^{\prime}(\xi)$. $a_{i}, b_{i}, c_{i}, d_{i}, N$ are constants to be determined later. $F(\xi)$ and $F^{\prime}(\xi)$ functions in Eq. (4) satisfy,

$$
F^{\prime 2}(\xi)=P F^{4}(\xi)+Q F^{2}(\xi)+R
$$

and therefore,

$$
\left\{\begin{array}{l}
F^{\prime \prime}(\xi)=2 P F^{3}(\xi)+Q F(\xi), \\
F^{\prime \prime \prime}(\xi)=\left(6 P F^{2}(\xi)+Q\right) F^{\prime}(\xi), \\
F^{(v)}(\xi)=24 P^{2} F^{5}(\xi)+20 P Q F^{3}(\xi)+\left(Q^{2}+12 P R\right) F(\xi), \\
F^{(5)}(\xi)=\left(120 P^{2} F^{4}(\xi)+60 P Q F^{2}(\xi)+Q^{2}+12 P R\right) F^{\prime}(\xi),
\end{array}\right.
$$

where $P, Q$ and $R$ are all parameters. Firstly, the number $N$ in the solution function must be determined.

The balancing numbers $N$ is positive integer which can be appointed by balancing the highest order derivative terms with highest power nonlinear terms in Eqs. (3). We substitute Eq. (4) and Eq. (6) into Eq. (3) and then to computerize a symbolic computation, equating to zero the coefficients of all power,

$F^{\prime i}(\xi) F^{j}(\xi) \quad(i=0,1 ; j=0, \mp 1, \mp 2, \cdots)$ yields a set of algebraic equations for $a_{i}, b_{i}, c_{i}, d_{i}$ and $\xi$.

\section{Application to Sine-Gordon Equation}

In this section, we apply our proposed method to nonlinear sine-Gordon equation [19]

$$
u_{t t}-u_{x x}+\sin (u)=0 \text {. }
$$

The sine-Gordon equation is one of the most important equation in many scientific fields such as the propagation of fluxons, solid state physics, nonlinear optics, and dislocations in metals. In order to implement new type of generalized F-expansion method, we introduce the transformation,

$$
v=e^{i u},
$$

and hence,

$$
\sin (u)=\frac{v^{2}-1}{2 i v},
$$

and also gives,

$$
u=\arccos \left(\frac{v^{2}+1}{2 v}\right) .
$$

Applying these transformations to the equation, then we have a differential equation,

$$
2 v v_{t t}-2 v v_{x x}-2 v_{t}^{2}+2 v_{x}^{2}+v^{3}-v=0
$$

Let we acquaint the following transformation,

$$
v(x, t)=\phi(\eta), \quad \eta=x-c t,
$$

where $C$ as the wave speed. Therefore Eq. (11) can be coverted to the ODE,

$$
2\left(c^{2}-1\right) \phi \phi^{\prime \prime}+2\left(1-c^{2}\right)\left(\phi^{\prime}\right)^{2}+\phi^{3}-\phi=0,
$$

where prime denotes the derivative with respect to $\eta$. In order to get $N$, when we apply balance procedure, $N=2$ is obtained. So, Jacobi elliptic functions solutions of Eq. (7) as follows:

$$
\begin{aligned}
& \phi(\eta)=a_{0}+a_{1} F(\eta)+a_{2} F^{2}(\eta)+\frac{b_{1}}{F(\eta)}+\frac{b_{1}}{F^{2}(\eta)} \\
& +c_{1} \frac{F^{\prime}(\eta)}{F(\eta)}+c_{2}\left(\frac{F^{\prime}(\eta)}{F(\eta)}\right)^{2}+d_{1} \frac{F(\eta)}{F^{\prime}(\eta)}+d_{2}\left(\frac{F(\eta)}{F^{\prime}(\eta)}\right)^{2} .
\end{aligned}
$$

Taking into consideration Eq. (5), Eq. (6) and substituting Eq. (14) into Eq. (13), we get system of algebraic equations. When this system is solved by the appropriate method, the desired coefficients are found. If we select $F(\eta)=s n(\eta)$ from Eq. (5), we take $P=m^{2}$, $Q=-\left(1+m^{2}\right)$ and $R=1$. In this case the following results are obtained. 


\section{Case 1:}

$$
\begin{aligned}
& a_{0}=\left(1+m^{2}\right) c_{2}, a_{1}=b_{1}=c_{1}=d_{1}=d_{2}=0, \\
& a_{2}=\left(m-m^{2}\right) c_{2}, b_{2}=-c_{2}, c= \pm \sqrt{\frac{4 m-1}{4 m}} .
\end{aligned}
$$

When the obtained coefficients are written in the solution function and the necessary transformations are applied, the solution of Eq. (7) is obtained as follows:

$$
u_{1}(x, t)=\arccos \left(A_{0}+A_{1} s n^{2}\left(\eta_{1}\right)-n s^{2}\left(\eta_{1}\right)+c s^{2}\left(\eta_{1}\right) d n^{2}\left(\eta_{1}\right)\right) \text {, }
$$

where $c_{2}=1, A_{0}=\left(1+m^{2}\right), A_{1}=\left(m-m^{2}\right)$, and

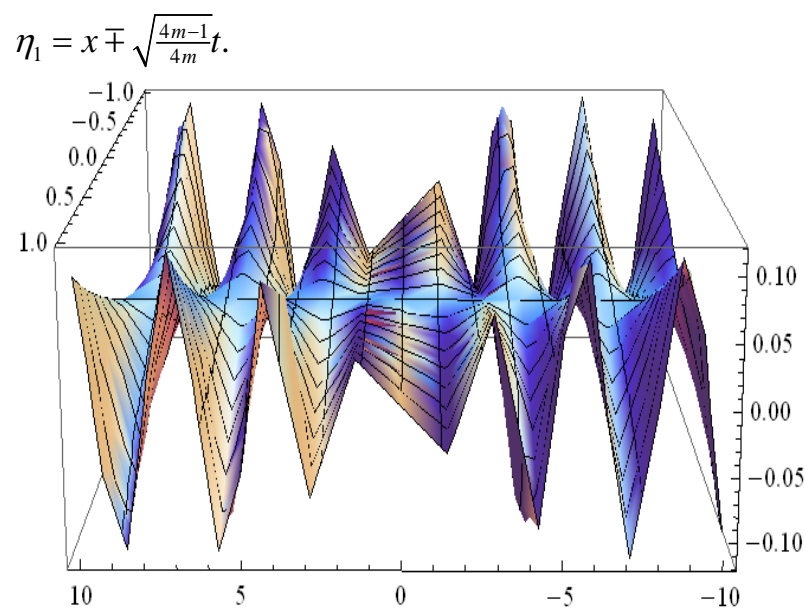

Figure 1. Graph of imaginary values of $u_{1}(x, t)$ in Eq. (16) is demonstrated at $m=\frac{1}{5}, c_{2}=12$.

\section{Case 2:}

$$
\begin{aligned}
& a_{0}=\frac{1+m^{2}+\left(4 m+4 m^{3}\right) c_{2}}{4 m}, a_{1}=b_{1}=c_{1}=d_{1}=0, \\
& a_{2}=\frac{m-4 m^{2} c_{2}}{4}, b_{2}=\frac{1-4 m c_{2}}{4 m}, \\
& d_{2}=\frac{\left(1+m^{2}\right)^{2}-4 m^{2}}{4 m}, c= \pm \sqrt{\frac{16 m-1}{16 m}}
\end{aligned}
$$

If we write Eq. (17) into the Eq. (14) we find combined Jacobi eliptic function solution as follows:

$$
\begin{aligned}
& u_{2}(x, t)=\arccos \left(\begin{array}{l}
A_{2}+A_{3} s n^{2}\left(\eta_{2}\right)+A_{4} n s^{2}\left(\eta_{2}\right) \\
\left.+A_{5} c s^{2}\left(\eta_{2}\right) d n^{2}\left(\eta_{2}\right)+\frac{s c^{2}\left(\eta_{2}\right)}{d n^{2}\left(\eta_{2}\right)}\right), \quad(18)
\end{array}\right. \\
& \text { where } \quad c_{2}=1, \quad A_{2}=\frac{4 m^{3}+m^{2}+4 m+1}{4 m}, \quad A_{3}=\frac{m-4 m^{2}}{4},
\end{aligned}
$$

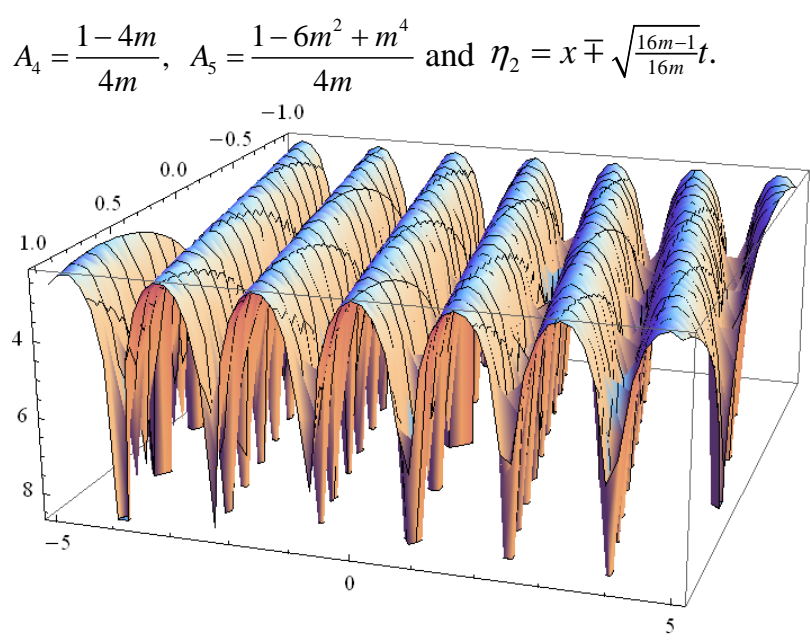

Figure 2. Graph of imaginary values of $u_{2}(x, t)$ in Eq. (18) is represented at $m=\frac{1}{5}, c_{2}=2$.

\section{Conclusions}

The new type of generalized F-expansion method is presented for finding more Jacobi elliptic function solutions of the sine-Gordon equation. This proposed method be newly submitted to literature. As a result, new and different types of solutions are acquired including multiple and the combined nondegenerative Jacobi elliptic function solutions. Three dimensional graphs of the obtained solutions are drawn according to the appropriate parameter values. More nonlinear differential equations can be solved by use of this new method.

\section{Referanslar}

1. Wang, M, Li, X, Zhang, J, The $G^{\prime} / G$-expansion method and travelling wave solutions of nonlinear evolution equations in mathematical physics, Physics Letters A, 2008, 372, 417-423.

2. He, J.H, Wu, X.H, Exp-function method for nonlinear wave equations, Chaos Solitons Fractals, 2006, 30, 700-708.

3. Hu, X.B, Ma, W.X, Application of Hirota's bilinear formalism to the Toeplitz lattice-some special soliton-like solutions, Physics Letters A, 2002, 293, 161-165.

4. Liu, C.S, Trial equation method for nonlinear evolution equations with rank inhomogeneous: mathematical discussions and applications, Сотmunications in Theoretical Physics, 2006, 45(2), 219-223.

5. Zhang, Y, Lie symmetry analysis and exact solutions of the SharmaTasso-Olever equation, IAENG International Journal of Applied Mathematics, 2016, 46(2), 1-5.

6. Pandir, Y, Gurefe, Y, Kadak, U, Misirli, E, Classifications of exact solutions for some nonlinear partial differential equations with generalized evolution, Abstract and Applied Analysis, 2012, 2012, 16 pp.

7. Chen, H.T, Hong-Qing, Z, New double periodic and multiple soliton solutions of the generalized (2+1)-dimensional Boussinesq equation, Chaos Solitons Fractals, 2004, 20, 765-769. 
8. Chen, Y, Yan, Z, The Weierstrass elliptic function expansion method and its applications in nonlinear wave equations, Chaos Solitons Fractal, 2006, 29, 948-964.

9. Abdou, M.A, Further improved F-expansion and new exact solutions for nonlinear evolution equations, Nonlinear Dynamics, 2008, 52, 227288.

10. Cai, G, Wang, Q, Huang, J, A modified F-expansion method for solving breaking soliton equation, International Journal of Nonlinear Science, 2006, 2, 122-128.

11. Zhang, S, Xia, T, A generalized F-expansion method with symbolic computation exactly solving Broer-Kaup equations, Applied Mathematics and Computation, 2007, 189, 949-955.

12. Zhang, $\mathrm{S}, \mathrm{Xia}, \mathrm{T}, \mathrm{An}$ improved generalized F-expansion method and its application to the $(2+1)$-dimensional KdV equations, Communications in Nonlinear Science and Numerical Simulation, 2008, 13, 1294-1301.

13. Demiray, S.T, Pandir, Y, Bulut, $H$, New solitary wave solutions of Maccari system, Ocean Engineering, 2015, 103, 153-159.
14. Pandir, Y, Symmetric Fibonacci function solutions of some nonlinear partial differential equtions, Applied Mathematics \& Information Sciences, 2014, 8(5), 2237-2241.

15. Ding, Y, He, B, Li, W, A improved F-expansion method and its application to the Zhiber-Shabat equation, Mathematical Methods in the Applied Sciences, 2012, 35(4), 466-473.

16. Wang, M, Li, X, Extended F-expansion method and periodic wave solutions for the generalized Zakharov equations, Physics Letters A, 2005, 343(1-3), 48-54.

17. Zhang, S, Xia, T.C, A generalized F-expansion method and new exact solutions of Konopelchenko-Dubrovsky equations, Applied Mathematics and Computations, 2006, 183(2), 1190-1200.

18. Ren, Y.J, Zhang, H.Q, A generalized F-expansion method to find abundant families of Jacobi Elliptic Function solutions of the (2+1)-dimensional Nizhnik-Novikov-Veselov equation, Chaos Solitons Fractals, 2006, 27(4), 959-979.

19. Wazwaz, A.M, The tanh method: exact solutions of the sine-Gordon and the sinh-Gordon equations, Applied Mathematics and Computations, 2005, 1677, 1196-1210. 